\title{
Direct interband light absorption in strongly oblate semiellipsoidal quantum dots' ensemble
}

\author{
David B. Hayrapetyan ${ }^{\mathrm{a}, \mathrm{b}, 1}$ \\ ${ }^{a}$ Russian-Armenian State University, 123 Hovsep Emin Str., Yerevan, 0051, Armenia. E-mail; \\ ${ }^{\mathrm{b}}$ State Engineering University of Armenia, \\ 105 Terian Str., Yerevan, 0009, Armenia.
}

\begin{abstract}
Within the framework of adiabatic approximation the energy levels and direct interband light absorption in strongly oblate semiellipsoidal quantum dot's ensemble are studied. Analytical expressions for the particle energy spectrum and absorption threshold frequency in the regime of strong size quantization are obtained. Selection rules for quantum transitions are revealed. To facilitate the comparison of obtained results with the probable experimental data, the small semiaxe size dispersion distribution of quantum dots growing by two experimentally realizing distribution functions have been taken into account. Distribution functions of Lifshits-Slezov and Gaussian have been considered.
\end{abstract}

Keywords: Oblate semiellipsoidal quantum dot, interband light absorption, selection rules, quantum dot ensemble

\section{INTRODUCTION}

Development of the novel growth techniques, such as the Stranski-Krastanov epitaxial method etc., makes possible to grow semiconductor quantum dots $(Q D s)$ of various shapes and sizes ${ }^{1-3}$. As is known, the energy spectrum of charge carriers $(C C s)$ in $Q D s$ is completely quantized and resembles the energy spectrum of atoms ("artificial atoms"). In a number of papers it has been shown, that small change in external shape of $Q D$ strongly influences energy spectrum and other characteristics of such semiconductor structures ${ }^{4}$. As a result of diffusion, the confining potential, formed during the growth process, in most cases can be approximated with a high accuracy by a parabolic potential. However, an effective parabolic potential may arise in a $Q D$ in view of features of its external shape ${ }^{5,6}$.

Theoretical investigations of optical properties of $Q D s$ remain to be a central concern of physicists because the results of these investigations can find direct applications in semiconductor devices of new generation ${ }^{7}$. Investigations of the optical absorption spectrum of various semiconductor structures are a powerful tool for determination of many characteristics of these systems: forbidden band gaps, effective masses of electrons and holes, their mobilities, dielectric permittivities, etc. There are many works devoted to the theoretical and experimental study of the optical absorption both in massive semiconductors and size-quantized systems. The presence of SiQ essentially influences the absorption mechanism. In fact, the formation of new energy levels of the $S Q$ makes possible new interlevel transitions.

From the theoretical point of view, spherical $Q D s$ are easier to investigate taking into account their symmetry, which allows to obtain analytical solutions for the energy spectrum, coefficient of absorption, charge carriers mobility, etc. ${ }^{8,9}$. However, modern methods for semiconductor nanostructures growth makes it possible to obtain $Q D s$ of different geometrical shapes and sizes. By now $Q D s$ of different geometrical forms and sizes are realized and studied theoretically: spherical, cylindrical, pyramidal, lens shaped, ellipsoidal, etc. ${ }^{10-15}$. Other examples of the $Q D s$ are semiellipsoidal $Q D s$. The advantage of these ones is the possibility of controlling the energy spectrum with two geometrical parameters (two semiaxes).

In this paper the electron states and direct interband absorption of light in a strongly oblate semiellipsoidal $Q D$ ( $S O S Q D$ ) is considered. Absorption edge and absorption coefficient are also considered. To facilitate the comparison of obtained results with the probable experimental data, the small semiaxe size dispersion distribution of quantum dots growing by two experimentally realizing distribution functions have been taken into account. Distribution function of Lifshits-Slezov has been considered in the first model and distribution function of Gauss has been considered in the second case.

${ }^{1}$ dhayrap82@gmail.com

Photonics and Micro- and Nano-structured Materials 2011, edited by Rafael Kh. Drampyan,

Proc. of SPIE Vol. 8414, 84140N · @ 2012 SPIE · CCC code: 0277-786X/12/\$18 · doi: 10.1117/12.923326 


\section{THEORY}

Let us consider an opaque strongly oblate semiellipsoidal quantum dot (Fig. 1 a)). Then the potential energy of a particle (electron, hole, exciton) in the cylindrical coordinates can be presented in the form

$$
U(X, Y, Z)=\left\{\begin{array}{l}
0, \frac{X^{2}+Y^{2}}{a_{1}^{2}}+\frac{Z^{2}}{c_{1}^{2}} \leq 1, \cap Z \geq 0 \\
\infty, \frac{X^{2}+Y^{2}}{a_{1}^{2}}+\frac{Z^{2}}{c_{1}^{2}}>1, \cap Z<0
\end{array} \quad Z \in\left[0, c_{1} \sqrt{1-\frac{\rho^{2}}{a_{1}^{2}}}\right], \quad c_{1} \ll a_{1}\right.
$$

where $c_{1}$ and $a_{1}$ are, respectively, the small and large semiaxes of the SOSQD.

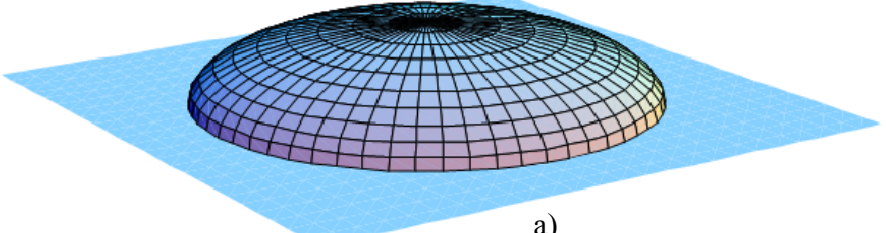

a)

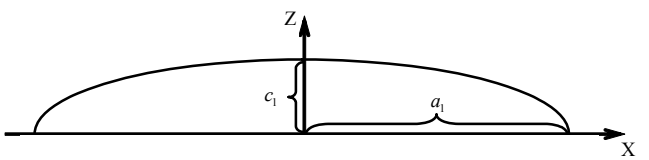

b)

Fig. 1. Strongly oblate semiellipsoidal quantum dot.

a) $3 \mathrm{D}$ picture, b) cross section

In the regime of strong $S Q$ the energy of the Coulomb interaction between an electron and hole is much less than the energy determined by the walls of the SOSQD. In this approximation the Coulomb interaction can be neglected. Then the problem is reduced to the determination of individual energy states of an electron and hole. From the geometrical form of a $Q D$ it follows that the particle motion along the Z-direction is faster than in the plane perpendicular to it. This allows one to use the adiabatic approximation ${ }^{16}$. The Hamiltonian of this system in the cylindrical coordinates has the form

$$
\hat{\mathrm{H}}=-\frac{\hbar^{2}}{2 m^{*}}\left\{\frac{\partial^{2}}{\partial \rho^{2}}+\frac{1}{\rho} \frac{\partial}{\partial \rho}+\frac{1}{\rho^{2}} \frac{\partial^{2}}{\partial \varphi^{2}}\right\}-\frac{\hbar^{2}}{2 m^{*}} \frac{\partial^{2}}{\partial Z^{2}}+U
$$

It can be represented as a sum of the Hamiltonians of the "fast" $\left(\hat{H}_{1}\right)$ and "slow" $\left(\hat{H}_{2}\right)$ subsystems in dimensionless quantities:

$$
\hat{H}=\hat{H}_{1}+\hat{H}_{2}+\hat{U}(r, \varphi, z)
$$

where

$$
\begin{gathered}
\hat{H}_{1}=-\frac{\partial^{2}}{\partial z^{2}} \\
\hat{H}_{2}=-\left[\frac{\partial^{2}}{\partial r^{2}}+\frac{1}{r} \frac{\partial}{\partial r}+\frac{1}{r^{2}} \frac{\partial^{2}}{\partial \varphi^{2}}\right]
\end{gathered}
$$

Here $\hat{H}=\frac{\hat{\mathrm{H}}}{E_{R}}, r=\frac{r}{a_{\mathrm{n}}}, z=\frac{Z}{a_{B}}$, e and $m_{p}$ are, correspondingly, the charge and effective mass of a particle (electron, hole, exciton), $E_{R}=\frac{\hbar^{2}}{2 m^{*} a_{B}^{2}}$ is the effective Rydberg energy, $a_{B}=\frac{\kappa \hbar^{2}}{m^{*} e^{2}}$ is the effective Bohr radius of a particle, $\kappa$ is the dielectric permittivity. The wave function is sought in the form 


$$
\psi(r, \varphi, z)=e^{i m \varphi} \chi(z ; r) R(r)
$$

At a fixed value of the coordinate $r$ of the slow subsystem the particle motion is localized in a one-dimensional potential well with the effective width (Fig. 1 b))

$$
L(r)=c \sqrt{1-\frac{r^{2}}{a^{2}}}
$$

where $a=\frac{a_{1}}{a_{B}}$ and $c=\frac{c_{1}}{a_{B}}$. From the solution of the Schrödinger equation for the first subsystem we obtain for the energy spectrum of a particle

$$
\varepsilon_{1}(r)=\frac{\pi^{2} n^{2}}{L(r)^{2}}, \quad n=1,2, \ldots
$$

Here $n$ is quantum number of the "fast" subsystem. For the low levels of the spectrum a particle is localized mainly in the region $r=a$. Based on this one can expand $e_{1}(r)$ into a series

$$
\varepsilon_{1}(r) \simeq \alpha_{n}+\beta_{n}^{2} r^{2}
$$

where $\alpha_{n}=\frac{\pi^{2} n^{2}}{c^{2}}, \beta_{n}=\frac{\pi n}{a c}$. Expression (9) is the effective potential entering the Schrödinger equation of the slow subsystem. For the total energy and the wave function of the system finally we get

$$
\begin{gathered}
\varepsilon=\alpha_{n}+2 \beta_{n}(N+1), \quad N=0,1,2, \ldots \\
\psi(r, \varphi, z)=\frac{e^{i m \varphi}}{\sqrt{2 \pi}} \sqrt{\frac{2}{z(r)}} \sin \left(\frac{\pi n}{z(r)} z+\pi n\right) \sqrt{2 \beta_{n}} \frac{\sqrt{n_{r} !}}{\Gamma^{1 / 2}\left(n_{r}+|m|+1\right)} e^{-\frac{\beta_{n} r^{2}}{2}}\left(\beta_{n} r^{2}\right)^{\frac{|m|}{2}} L_{n_{r}}^{|m|}\left(\beta_{n} r^{2}\right)
\end{gathered}
$$

Here $N$ is oscillatory quantum number, $m$ is magnetic quantum number, $n_{r}$ is radial quantum number, $L_{n_{r}}^{|m|}$ is Laguerre polynomials.

\section{DIRECT INTERBAND LIGHT ABSORPTION}

We proceed to the consideration of the direct interband light absorption in an SOSQD in the regime of strong $S Q$, when the Coulomb interaction between an electron and hole can be neglected. Let us consider the case of a heavy hole, when $m_{e}^{*} \ll m_{h}^{*}$. The absorption coefficient is defined by the expression

$$
K=A \sum_{\nu, \nu^{\prime}}\left|\int \Psi_{\nu}^{e} \Psi_{\nu^{\prime}}^{h} d \vec{r}\right|^{2} \delta\left(\hbar \Omega-E_{g}-E_{\nu}^{e}-E_{\nu^{\prime}}^{h}\right)
$$

where $\nu$ and $\nu^{\prime}$ are the sets of quantum numbers $(Q N s)$ corresponding to an electron and heavy hole, $E_{g}$ is the forbidden band width of a massive semiconductor, $\Omega$ is the frequency of the incident light, A is a quantity proportional to the square of the matrix element taken by the Bloch functions ${ }^{17}$. Finally, in the regime of strong $S Q$, for the quantity $K$ and absorption edge $(A E)$ we obtain

$$
\begin{gathered}
K=A \sum_{n, m, N} \delta\left(\hbar \Omega-E_{g}-E_{e}-E_{h}\right) \\
W_{100}=1+\pi^{2} \frac{d^{2}}{c_{1}^{2}}+2 \pi \frac{d^{2}}{a_{1} c_{1}}
\end{gathered}
$$

where $W_{100}=\frac{\hbar \Omega_{100}}{E_{g}}$ and $d=\frac{\hbar}{\sqrt{2 \mu E_{g}}}$. Formula (14) characterizes the dependence of the effective forbidden band width 
on the semiaxes $a_{1}$ and $c_{1}$. With the decrease in the both semiaxes the $A E$ shifts to the short-wave region, but the dependence on the small semiaxis is stronger. Consider now the selection rules for $Q N s$. For the magnetic $Q N$ the transitions between the levels with $m=-m^{\prime}$ are allowed, and for the $Q N$ of the first subsystem - the transitions with $n=n^{\prime}$. For the oscillatory $Q N$ the transitions for the levels with $N=N^{\prime}$ are allowed. Note that the analytic form of expression (13) is presented with allowance for the mentioned selection rules of $Q N S$.

So far we have studied the absorption of a system consisting of semiconductor $Q D s$ having identical dimensions. For comparison of the obtained results with experimental data, one has to take into account the random character of $S O S Q D$ dimensions (or half-axis) intrinsic for the growth process. The absorption coefficient should be multiplied by concentration of $Q D s$. Instead of distinct absorption lines, account of size dispersion will give a series of maximums. In the first model we use the Lifshits-Slezov distribution function?:

$$
P(u)=\left\{\begin{array}{l}
\frac{3^{4} e u^{2} \exp (-1 /(1-2 u / 3))}{2^{5 / 3}(u+3)^{7 / 3}(3 / 2-u)^{1 / 3}}, u<3 / 2 \\
0, u>3 / 2
\end{array}, u=\frac{c}{\bar{c}}=\frac{c_{1}}{\bar{c}_{1}}\right.
$$

where $\bar{c}$ is some average value of the half-axis. In the second model the Gaussian distribution function is used (see e.g. $\left.{ }^{18}\right)$ :

$$
P(u)=C e^{-\frac{(u-1)^{2}}{\sigma / \bar{c}}}
$$

Using these two distribution functions specifying the growth process can form two type $Q D s$ ensembles with symmetric and asymmetric distributions. Gaussian distribution describes the symmetric case and the Lifshits-Slezov distribution describes the asymmetric case. With a consideration of size distribution function the absorption coefficient is defined by the following expression

$$
K=A \sum_{m, n, N} \int_{0}^{3 / 2} P(u) d u \delta\left(\hbar \Omega-E_{g}-E_{\nu}^{e}-E_{\nu}^{h}\right)
$$

In the case of strong $S Q$ with account of general size distribution function $P(u)$ we obtain for the absorption coefficient corresponding formula:

$$
K=\frac{A}{E_{g}} \sum_{m, n, N} \frac{1}{\sqrt{\lambda_{3}^{2}+4 \lambda_{1} \lambda_{2}}}\left(\frac{2 \lambda_{2}}{\sqrt{\lambda_{3}^{2}+4 \lambda_{1} \lambda_{2}}-\lambda_{3}}\right)^{2} P\left(\frac{2 \lambda_{2}}{\sqrt{\lambda_{3}^{2}+4 \lambda_{1} \lambda_{2}}-\lambda_{3}}\right)
$$

where $\lambda_{1}=\frac{\hbar \Omega-E_{g}}{E_{g}}, \lambda_{2}=\frac{\pi^{2} n^{2}}{4}\left(\frac{d}{\bar{c}_{1}}\right)^{2}, \lambda_{3}=\pi n \frac{d^{2}}{a_{1} \bar{c}_{1}}(N+1)$.

\section{DISCUSSION}

As is seen from formula (10), the energy spectrum of CCs in a $S O S Q D$ is equidistant. This result is true only for the low levels of the spectrum (for small values of $Q N s$ ). Numerical calculations for the regime of strong $S Q$ were carried out for a GaAs quantum dot with the following parameters: $m_{e}^{*}=0.067 m_{e}, m_{e}^{*}=0.12 m_{h}^{*}, k=13.8$, $E_{R}=5.275 \mathrm{meV}$, effective Bohr radii of an electron and hole $a_{e}=104 \AA$ and $a_{h}=15 \AA, E_{g}=1.43 \mathrm{eV}$ is the forbidden band width of a massive semiconductor. In the regime of strong $S Q$ the frequency of the transition between the equidistant levels (for the value $\mathrm{n}=0$ ), for fixed values $a=2.5 a_{e}$ and $c=0.5 a_{e}$ is equal to $\omega_{10}=3.56 \times 10^{14} \mathrm{~s}^{-1}$, which

corresponds to the infrared region of the spectrum. At the same value of the $Q N$ but with $a=2 a_{e}$ and $c=0.4 a_{e}$ we 
have $\omega_{10}=5.56 \times 10^{14} \mathrm{~s}^{-1}$ which is 1.5 times as high as before. As is seen from formula (10), with increase of semiaxes lengths the particle energy decreases, and it is more "sensitive" to the variations of the small semiaxis, which is a consequence of the larger contribution of the $S Q$ into the particle energy in the direction of the semiellipsoid revolution. It should be noted that with the increase of semiaxes lengths the energy levels become closer together, but they remain equidistant.

Figures 2 and 3 present the dependences of the absorption threshold on the small and large semiaxes of an $S O S Q D$, respectively. With the decrease of semiaxes the absorption edge increases, which is a consequence of the increase in the $S Q$ (the "effective" forbidden band width increases). As is seen from the plots, the change in the absorption edge manifests itself clearly in the dependence on the small semiaxis. For the same reason the curves corresponding to different values of the small semiaxis (Fig. 2) provide a larger shift than in the opposite case (Fig. 3).

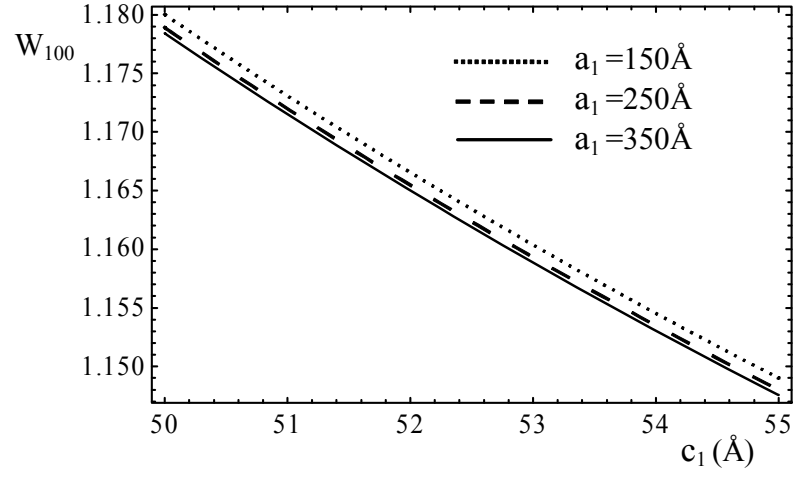

Fig. 2. Dependences of the absorption threshold on the small semiaxis of the $S O S Q D$ at a fixed value of the large semiaxis.

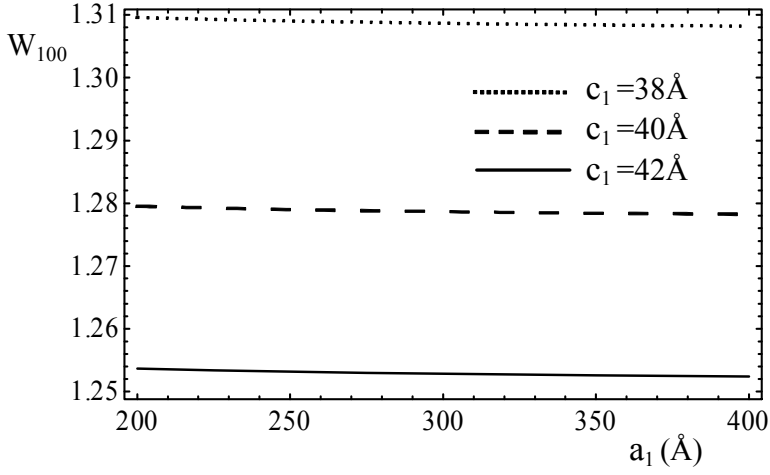

Fig. 3. Dependences of the absorption threshold on the large semiaxis of the $S O S Q D$ at a fixed value of the small semiaxis.

Fig.4 illustrates the dependence of absorption coefficient $K$ on the frequency of incident light for the first two equidistant families for the case of Gaussian distribution, when $\bar{c}_{1}=50 \AA$ and $a_{1}=250 \AA$. On the top of the right side of the figure the sum of the absorption coefficients corresponding to the separate transitions also is presented.

As it is mentioned above, instead of distinct absorption lines, consideration of size dispersion will give a series of maximums. Fig.5 illustrates the same dependence for the Lifshits-Slezov distribution case. Note that both in the model of Gaussian distribution and in the model of Lifshits-Slezov $Q D s$ distribution a single distinctly expressed maximum of absorption is observed. When the frequency of light is increased, the second weakly expressed maximum can be observed. Further increase of the incident light frequency results in a fall of absorption coefficient. Fig.6 illustrates the dependencies of the absorption coefficient on the frequency of incident light for both cases.

Schematic diagrams for appropriate transitions corresponding to light absorption are depicted on the Fig.7 to understand in detail the process of absorption. From the comparison of graphs on Fig.4 and Fig.5, it is obvious that pronounces maximum corresponds to the $n=n \phi=1$ transition family and weak expressed peaks are the result of the transitions between equidistant levels. The second weaker peak corresponds to the $n=n \phi=2$ transition family. It is also obvious from Fig.4 and Fig.5 that the intensity corresponding to the above mentioned family is weaker than the first maximum. This is the result of the small volume of the overlapping integral. The above mentioned means, that the probability of transition is decreased. 


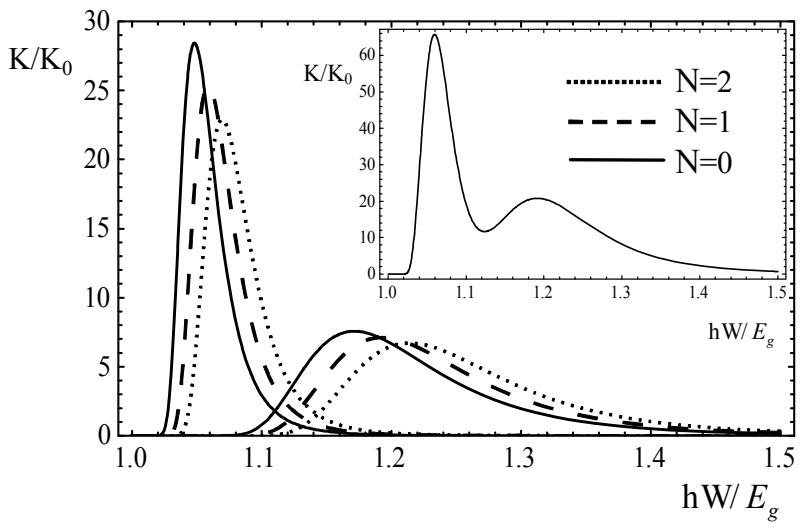

Fig. 4. Dependencies of absorption coefficient on the frequency of incident light for the first two equidistant families for the case of Gaussian distribution (The sum of absorption coefficient for all transitions).

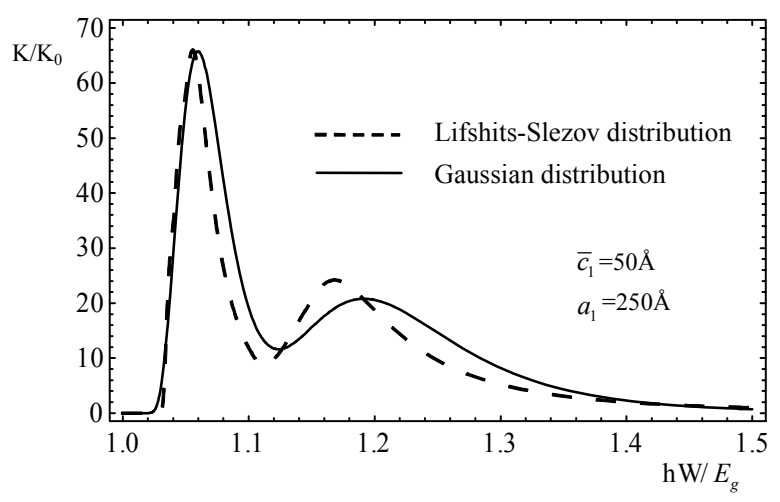

Fig. 6. Dependencies of the absorption coefficient on the frequency of incident light.

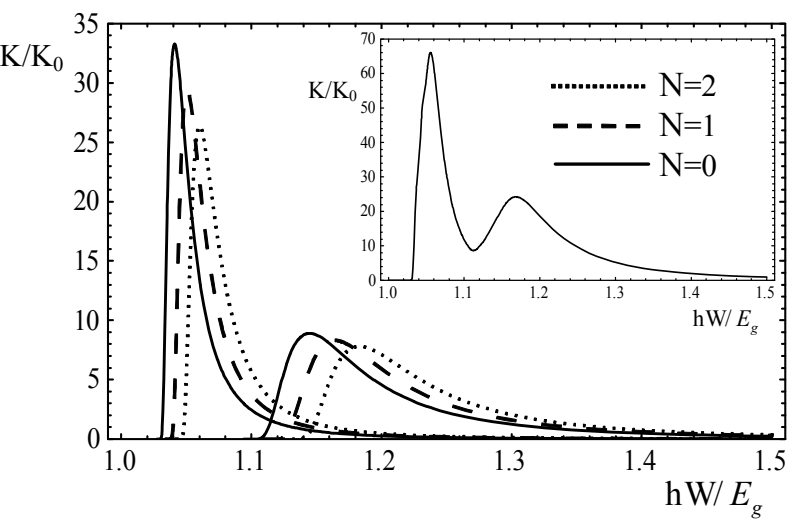

Fig. 5. Dependencies of the absorption coefficient on the frequency of incident light for the first two equidistant families for the case of Lifshits-Slezov distribution (The sum of absorption coefficient for all transitions).

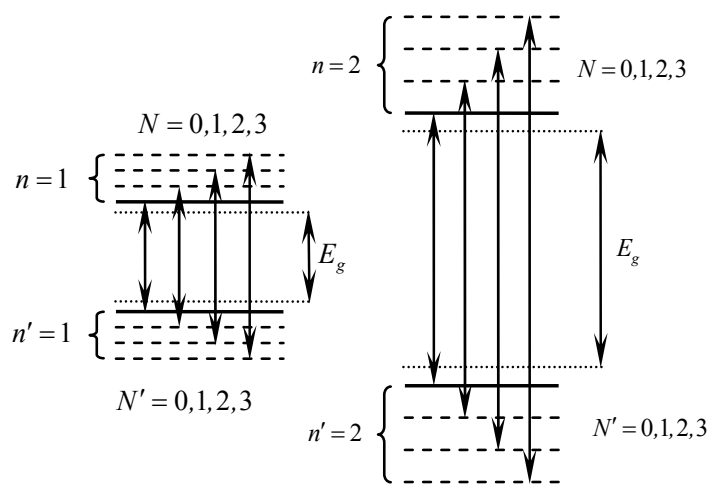

Fig.7. Schematic plot of corresponding interband transitions.

\section{CONCLUSION}

In this work obtained that the electron energy is equidistant inside $S O S Q D$ in the regime of strong $S Q$ for the low levels of the spectrum. The impact of the dispersion of geometrical sizes for the $Q D s$ ensemble on direct light absorption is also investigated. Selection rules for quantum transitions are revealed. This theoretical investigation of $S O S Q D s$ can be effectively used for direct applications in photonics as background for simulation model. For further investigations, it is also important to develop a scheme for optimization of growth of SOSQDs needed for second harmonic generation. 


\section{REFERENCES}

[1] Harrison, P., [Quantum Wells, Wires and Dots. Theoretical and Computational physics], Wiley, New York, (2005).

[2] Bastard, D., [Wave Mechanics Applied to Semiconductor Heterostructures], Les editions de physique, Paris, (1989).

[3] Kazaryan, E.M., Petrosyan S.G., [Physical Principles of Semiconductor Nanoelectronics], RAU, Yerevan, (2005).

[4] Dvoyan, K.G., Kazaryan, E.M., "Impurity states in a weakly prolate (oblate) ellipsoidal microcrystal placed in a magnetic field", Physica Status Solidi (B), 228, 695-703 (2001).

[5] Hayrapetyan, D.B., "Direct interband light absorption in a strongly prolated ellipsoidal quantum dot", Journal of Contemporary Physics, 42, 293-298 (2007).

[6] Hayrapetyan, D.B., Dvoyan, K.G., Kazaryan, E.M., "Direct interband light absorption in a strongly oblate ellipsoidal quantum dot", Journal of Contemporary Physics, 42, 151-157 (2007).

[7] Sze, S.M., [Semiconductor devices: Physics and technology], Wiley India Pvt. Ltd, (2009).

[8] Ramaniah, L.M., Nair, S.V., "Optical absorption in semiconductor quantum dots: A tight-binding approach", Phys. Rev. B 47, 7132-7139 (1993).

[9] Efros, Al. L. and Efros, A.L., "Interband absorption of light in a semiconductor sphere" Sov. Phys. Semicond., 16, 772-775 (1982).

[10] Dvoyan, K.G., Hayrapetyan, D.B., Kazaryan, E.M., Tshantshapanyan, A.A., "Electronic states and light absorption in a cylindrical quantum dot having thin falciform cross section", Nanoscale Research Letters, 4, 130-137 (2009).

[11] Sarkisyan, H.A. "Electronic states in a cylindrical quantum dot in the presence of parallel electrical and magnetical fields", Modern Physics Letters B, 16, 835-841 (2002).

[12] Aghekyan, N.G., Kazaryan, E.M., Petrosyan, L.S., Sarkisyan, H.A., "Two electron states in a quantum ring: Mathieu equation approach", Journal of physics: Conference Series, 248, 012048 (2010).

[13] Dvoyan, K.G., Hayrapetyan, D.B., Kazaryan, E.M., "Direct interband light absorption in strongly prolated ellipsoidal quantum dots ensemble", Nanoscale Research Letters, 4, 106-112 (2009).

[14] Hwang, T.M., Lin, W.W., Wang, W.Ch., Wang, W., "Numerical simulation of three dimensional pyramid quantum dot", Journal of Computational Physics, 196, 208-232 (2004).

[15] Sivakami, A., Mahendran, M., "Hydrostatic pressure and conduction band non-parabolicity effects on the impurity binding energy in a spherical quantum dot", Physica B: Physics of Condensed Matter, 405, 1403-1407 (2010).

[16] Galitsky, V.M., Karnakov, B.M., Kogan, V.I., [Practical Quantum Mechanics], Nauka, Moscow, (1981).

[17] Anselm, A.I., [Introduction to semiconductors theory], Nauka, Moscow, (1978).

[18]Kumar, A., Biswas, D., "Effects of a Gaussian size distribution on the absorption spectra of III-V semiconductor quantum dots", Journal of Applied Physics, 102, 084305-084305-5 (2007). 